\title{
La enseñanza de idiomas en España en la frontera de los años 30 : las ilusiones perdidas
}

Francisco José Morales Gil

\section{OpenEdition \\ Journals}

Edición electrónica

URL: https://journals.openedition.org/dhfles/785

DOI: $10.4000 /$ dhfles.785

ISSN: 2221-4038

Editor

Société Internationale pour l'Histoire du Français Langue Étrangère ou Seconde

Edición impresa

Fecha de publicación: 1 junio 2009

Paginación: 231-248

ISSN: 0992-7654

Referencia electrónica

Francisco José Morales Gil, « La enseñanza de idiomas en España en la frontera de los años 30 : las ilusiones perdidas », Documents pour l'histoire du français langue étrangère ou seconde [En ligne], 42 |

2009, mis en ligne le 16 janvier 2011, consulté le 14 juin 2021. URL : http://journals.openedition.org/ dhfles/785; DOl : https://doi.org/10.4000/dhfles.785

Este documento fue generado automáticamente el 14 junio 2021.

(c) SIHFLES 


\title{
La enseñanza de idiomas en España en la frontera de los años 30 : las ilusiones perdidas
}

\author{
Francisco José Morales Gil
}

\section{La educación en España en el primer tercio del siglo $\mathrm{XX}$}

1 El primer tercio del siglo XX fue crucial para España en todos los órdenes de la vida social y, por supuesto, también en el terreno educativo. Para García Hoz (1980 : 119), en las dos primeras décadas se habrían instalado, tanto los cimientos políticos y pedagógicos como los grandes problemas que se irían posteriormente desarrollando y confrontando a lo largo del siglo XX. Y es que, como señala Antonio Romero (1998: 28), la educación se va a convertir en estos años en el centro de un apasionado debate que trascenderá más allá de la escuela y en el que intervendrán intelectuales, grupos políticos, sociales, y, por supuesto, la Iglesia. El control de la educación será el objetivo último de esta pugna, y la iglesia católica, tradicional detentadora de un poder prácticamente omnímodo en la educación de los españoles ${ }^{1}$, verá levantarse frente a ella fuerzas de extraordinario empuje renovador, como la Institución Libre de Enseñanza, la Escuela Moderna, y en general todas las fuerzas liberales y progresistas. Frente a esta ofensiva, la Iglesia, que veía en peligro la enorme influencia que había conseguido ejercer sobre la sociedad española, « comienza a reorganizarse para dar la batalla en el terreno educativo» (Romero 1998: 30). En esta batalla, y siguiendo la llamada a la acción católica lanzada por el Papa León XIII en su encíclica Rerum Novarum, la iglesia española abrirá todos los frentes a su alcance, con el fortalecimiento de sus diferentes órdenes, la organización de congresos pedagógicos católicos y la creación de fundaciones y otros círculos para la « intervención social ».

Esta verdadera guerra entre la iglesia católica y las fuerzas progresistas alcanzará una virulencia inusitada, presagio de lo que se avecinaría, y no habrá terreno en el que no 
se entable batalla, ni siquiera la enseñanza del francés, como lo muestran estas palabras del activista Ruiz Amado (en Cuesta 1994 : 216) :

Hemos de protestar contra la vana 'moda', que considera el francés como elemento esencial de 'educación superior femenina' (...) leer libros franceses, no será, para las jóvenes, sino dañoso, las más de las veces; pues, no leerán seguramente a Bossuet o Bourdelone, ni siquiera a Fenelón, sino fútiles novelas y otras producciones frívolas de la más frívola de las Literaturas (...). Otro 'agravante' se añade, a las lenguas modernas indiscretamente enseñadas a las jóvenes, en el caso frecuente de que las hijas aprendan idiomas que ignoran sus madres, y por ventura sus padres. De lo cual han de resultar forzosamente peligros, situaciones tristemente cómicas para los padres, y menoscabo de su autoridad en el concepto de las hijas 'marisabidillas'.

3 Finalmente, observando los primeros 70 años del siglo XX, el periodo republicano quedará como un corto paréntesis en el predominio de la iglesia católica sobre la educación española.

Pero volviendo al terreno más propiamente pedagógico, según García Hoz (1980 : 216), serían Giner de los Ríos, a través de la Institución Libre de Enseñanza y Manjón, a través de las Escuelas del Ave María, cada uno a su manera, los impulsores en España de un tipo de enseñanza que más adelante se llamaría educación activa, o pedagogía intuitiva. Ambos, aunque discrepaban profundamente en su concepto de los fines de la educación, se oponían a la educación predominantemente 'libresca' de la época. A pesar de que la filosofía de las Escuelas del Ave María era más cercana a las ideas que sustentaban a la dictadura de Primo de Rivera, aquellas quedaron en una especie de aislamiento que minimizó su influencia en el contexto educativo. La Institución Libre de Enseñanza, sin embargo, realizó una labor mucho más eficaz. Si Giner de los Ríos tuvo en Cossío un continuador brillante, las Escuelas del Ave María empezaron a decaer con la muerte de su fundador en 1923.

5 Es necesario señalar igualmente en estos años la importante influencia de la pedagogía francófona en España : Ovide Decroly, de l'Ecole de l'Ermitage, que recibió en Bruselas a numerosos pedagogos españoles y visitaría Barcelona en 1921 y Madrid en 1926; el reconocido magisterio de Edouard Claparède y Adolphe Ferrière (y más tarde de Piaget), del Instituto Rousseau de Ginebra; o las influencias de Roger Cousinet y de Freinet, que dictó tres lecciones en la Escola d'Estiu de Barcelona en 1933 y cuyo método se propagó rápidamente por España (Monés 1999 : 29).

Por otra parte, la educación secundaria española, con el plan de 1903, había entrado en un periodo de estabilidad que duraría hasta 1926. Por el contrario, la llegada de la República traerá consigo, como en tantos terrenos, grandes proyectos de transformación del sistema educativo en general y de la segunda enseñanza en particular.

7 Los principios educativos republicanos eran pues deudores de la I.L.E. por un lado, y de las corrientes pedagógicas más avanzadas que circulaban por Europa y los Estados Unidos desde finales del siglo XIX : una escuela modernizadora, influyente en el cambio social ; activa, intuitiva y lúdica, en lo pedagógico ; tolerante desde el punto de vista político; laica e independiente frente al poder de la Iglesia, e igualitaria frente a la discriminación de la mujer (Domínguez 1999 : 45-47). A todo ello habría que añadir un formidable esfuerzo en construcciones escolares habida cuenta de las múltiples carencias a las que la República hubo de hacer frente de forma urgente (Puelles 1986: 347). 


\section{La enseñanza de las lenguas vivas : panorama internacional}

8 Coincidiendo con los últimos años del siglo XIX y con la revolución directa ${ }^{2}$, en plena corriente favorable a todo lo concerniente a la enseñanza de las lenguas vivas, se empiezan a constituir las primeras asociaciones de profesores (Martínez de Blanco 1962). En 1909 la Association de Professeurs de Langues Vivantes francesa convocará en París el primer Congreso Internacional, al que asistirían 451 congresistas de veinte países diferentes. España, carente de asociación, estuvo representada a título individual por Américo Castro. Recordemos que un año antes, en 1908, ya se habían revisado en Francia algunos de los postulados que habían constituido el "golpe de estado" pedagógico de los métodos directos en 1902. El método directo ya nunca más volvería a recuperar el poder que tuvo en esos primeros años del siglo XX (Puren 1988 : 99).

9 Sin embargo, en los años veinte y treinta se asistía, una vez pasada la fiebre directa, a un cierto decaimiento del entusiasmo despertado unos años antes por la enseñanza de los idiomas. Para Fernand Mossé (1960) una de las causas principales de este paréntesis había sido el aumento del número de alumnos por clase ${ }^{3}$, que había dificultado aún más la puesta en práctica de los nuevos métodos y producido un progresivo deterioro en el ánimo de los profesores. Este relativo decaimiento coincidirá con un periodo de eclecticismo metodológico, como testimoniaba Albert Séchehaye (1921: 461) :

L'époque est révolue du conflit aigu entre la méthode ancienne et la méthode directe dans l'enseignement des langues modernes. D'une manière de plus en plus générale, on adopte une sorte de compromis, d'ailleurs parfaitement légitime, entre les deux écoles, et l'on procède à une initiation grammaticale méthodique au cours d'exercices gradués dont la forme s'inspire de la méthode directe. Ce procédé mixte, fécondé par des apports accessoires empruntés aux recherches de Gouin ou de Carré, mis au point conformément aux exigences de la psychologie moderne peut fournir de bons résultats.

Existía así un amplio consenso sobre una metodología 'mixta' que finalmente perdurará, con diferentes matices, hasta principios de los sesenta. Tanto es así que Séchehaye se quejaba de que el único problema que parecía existir era " cette question toute pratique de savoir comment on peut assurer partout les conditions extérieures nécessaires au succès : bon manuel, maître bien préparé, nombre d'heures suffisant, classes pas trop nombreuses, etc. » (1921: 461).

11 El metodólogo alemán Adolf Bohlen llegará a afirmar que lo característico de este periodo de la enseñanza de las lenguas vivas es « el paulatino abandono de una meta predominantemente utilitaria, y la consecuente incorporación progresiva de la enseñanza de los idiomas la misión formativa general de la Enseñanza Media » (Bohlen $1963: 20)$.

12 Gracias a la encuesta llevada a cabo por el Bureau International d'Éducation, preparatoria de la VI Conferencia Internacional sobre la Instrucción Pública (Ginebra 1937) y consagrada a la enseñanza de las lenguas vivas, disponemos de una información detallada de la situación de la enseñanza de las lenguas vivas en estos años. Al objeto de poder situar con referencias válidas las condiciones que se daban en nuestro país, 
resumiremos brevemente a continuación los aspectos más relevantes y las tendencias generales (BIE 1937 : 9-40):

- Las lenguas vivas se estudiaban generalmente en todos los centros en los que se impartía cualquier modalidad de estudios secundarios, en las Escuelas Normales y Escuelas Profesionales. En muchos países se enseñaban también en las escuelas primarias, aunque de manera muy irregular y nunca de manera obligatoria.

- Lenguas más enseñadas : el francés, el inglés y el alemán (por ese orden).

- Objetivos asignados a estas enseñanzas : práctico, cultural, formativo y para fomentar la comprensión entre los pueblos.

- Métodos de enseñanza : el método más generalizado, siempre dentro de una gran libertad metodológica, es el llamado mixto o " modéré ».

- Los manuales : suelen ser controlados por comisiones técnicas dependientes de los ministerios.

En lo que se refiere a nuestro país, por una vez, gracias a las instrucciones republicanas de 1934, estábamos perfectamente integrados (a mediados de los años treinta) en las corrientes internacionales más modernas.

\section{Las lenguas vivas en el sistema educativo español}

Desde su aparición en el sistema educativo, las lenguas vivas habían recibido una acogida ciertamente desdeñosa por parte de los representantes de las materias tradicionalmente bien instaladas y que se sentían ajenas a cualquier necesidad de justificar su dominante presencia en el mismo. Esto se debió, como señalaba Pablo Sicart (1947 : 11), a que se consideraban estos estudios como cursos de adorno faltos de valor educativo. Su carácter de enseñanza práctica y su utilidad inmediata la alejaba de las grandes asignaturas clásicas, cultivadas casi exclusivamente por su valor formativo y sin necesidad alguna de demostrar la más mínima utilidad a corto plazo. A lo dicho se le sumaba el menosprecio hacia un profesorado cuya formación era indudablemente muy diversa, y a quien la administración marginaba al mantenerlo oficial y oficiosamente en la categoría de los "profesores especiales". Y ello en todos los niveles educativos, empezando por su cúpula, las universidades, como señalaba Américo Castro en un interesantísimo artículo publicado en1921 en el Boletín de la I.L.E. : « en España las Universidades desconocen el cultivo de las lenguas modernas » (Castro 1921 : 13).

Se lamentaba Castro de la inexistencia en nuestro país, ni aún en la Facultad de Letras, de estudios sobre civilizaciones y literaturas extranjeras, así como de la incapacidad de los alumnos universitarios para enfrentarse a textos en inglés o en alemán, y que sólo alguno «maltraduce » el francés. Reivindicaba para la Facultad de Letras de Madrid una « decorosa » enseñanza del francés, aunque sólo fuese para influir en la formación de los profesores de instituto, a los que en aquella época no se exigía título universitario alguno ${ }^{4}$. El problema empezaba en los institutos, en los que el estudio de las lenguas modernas debía facilitar a los bachilleres una preparación que les sirviera para sus viajes o para formarles en eventuales relaciones comerciales, científicas, etcétera. 0 "sencillamente para ser un átomo más en ese ambiente de cultura media, que en último término es el que da el tono a los países civilizados » (Castro 1921 : 120).

Sin embargo, la situación de la enseñanza de las lenguas vivas en España en los niveles educativos no universitarios era dibujada por Castro como catastrófica ${ }^{5}$ : 
En este punto no hemos rebasado el plano más inferior, en que comienza el interés por aquellos idiomas; se considera su estudio como un instrumento útil o de adorno. Los españoles, abandonados a sí mismos, los aprenden aisladamente, cuando la necesidad les apremia ; el estado se limita a mantener una apariencia de enseñanza en los Institutos (dos años de francés, un año de inglés o alemán) y Escuelas de Comercio. ¿Quién ignora, empero, que nadie aprende a hablar y escribir correctamente una lengua en esos Centros? Es decir, que ni aún la finalidad más modesta y elemental se logra dentro de nuestra enseñanza (Castro 1921 : 120).

Y es que, a pesar de las repetidas llamadas del Ministerio a favor de una enseñanza más práctica, el profesorado se había ido acomodando a una concepción formativo-cultural asentada en métodos tradicionales completamente ajenos a cualquier fin utilitario de la lengua, y muy pocos profesores se habían atrevido a intentar poner en práctica en sus clases el método directo (Fernández Fraile 1996: 235-239). Castro, por su parte, dibujaba un panorama desolador, con clases de cien alumnos y consagradas al estudio memorístico de la gramática :

Recuerdo mi propia experiencia: en el segundo año de francés 'se daba' la gramática en francés; es decir, que nos aprendíamos de memoria los capítulos de un perverso manual y con tono decidido declamábamos aquellas tonterías : « Outre les règles générales déjà établies... » Años más tarde, cuando supe francés, me apareció en la memoria ese guiñapito de cultura, y entonces me di cuenta que outre significa 'además de' (Castro 1921 : 121).

No obstante, antes de las reformas de 1926 sí se darían pasos importantes en el desarrollo de la enseñanza de los idiomas en España, como la creación en 1911 de la Escuela Central de Idiomas, la creación en tiempos del General Primo de Rivera de Institutos de Idiomas en muchas universidades (Canito $1952: 252$ ), o las esperanzadoras experiencias llevadas a cabo, desde 1918, en el Instituto-Escuela de Madrid. En esa misma línea de avances cabe señalar que el francés había entrado por fin en las Escuelas del Magisterio, aunque en la práctica, salvo lo relacionado con los tres contenidos clásicos - leer, escribir y contar - del resto de la formación que recibía el Maestro (Religión, Geografía, Historia, Ciencias Naturales, Dibujo, Gimnasia y Música) casi nada se proyectaría por ahora, de manera directa, en la escuela primaria.

\section{Las últimas reformas de la Monarquía}

Durante los años 1900-1930 la habitual agitación que envolvía a la 'segunda enseñanza' se calma y asistimos a una pausa legislativa que permite pasar un cuarto de siglo sin que se modifique el plan de estudios del bachillerato (Utande $1982: 19$ ). Finalmente, en 1926, un nuevo plan de estudios para la segunda enseñanza (Plan Callejo) acabaría con la excepcional estabilidad del plan de 1903.

En lo referente a las lenguas vivas, el correspondiente cuestionario oficial (R. O. de 9 de agosto de 1927) supondría la aparición de las primeras orientaciones metodológicas, documento que permite « calibrar el estado de la cuestión metodológica durante esos años » (Fernández Fraile 1996: 352). Efectivamente, dentro de un tono general muy dirigista y aunque lleno de aspectos confusos e incluso contradictorios, el cuestionario de 1927 se sitúa en las posiciones eclécticas dominantes en Europa en aquellos años. Recordemos que tras la revolución directa de principios de siglo (revolución que apenas existió en España), y la caída en desgracia de dichos métodos, una corriente revisionista se había instalado en los países de mayor tradición en nuestra disciplina, sobre todo en 
Francia, país en el que los principios directos se habían impuesto de una manera particularmente radical (Puren 1988). Por ello, las instrucciones españolas de 1927 entroncaban perfectamente con este cambio de rumbo, y no habiéndose conocido en nuestro país una situación similar a la de Francia con los métodos directos, este eclecticismo suponía en general - a pesar de la desfiguración del método directo - un verdadero progreso, fundamentalmente en cuanto al aprendizaje inductivo de la gramática y a la práctica oral (Fernández Fraile 1996 : 359-360).

21 Aún habría tiempo, antes del advenimiento de la República, de un nuevo Plan de estudios (20 de agosto de 1930), que daba un giro contrario al anterior, estableciendo un nuevo nombre para la asignatura, 'Lengua y Literatura francesa', y unos objetivos muy claros : traducción de textos y conocimientos literarios. Pero esta reforma tendría una vida extremadamente corta y escasas consecuencias.

5. República y enseñanza de Lenguas Vivas

Con la llegada de la República, la ola reformadora alcanzaría también a la enseñanza de las lenguas vivas, fundamentalmente en tres aspectos: las nuevas orientaciones metodológicas, la creación de la licenciatura de Filología Moderna (cuarenta y seis años más tarde que en Francia, por ejemplo) y la exigencia de ese título para poder presentarse a las oposiciones.

24 En 1931 el ministro Marcelino Domingo Sanjuán recurriría por un año al plan de 1903 « una de las disposiciones más juiciosas que la administración educativa ha tomado en España » (García Hoz 1980: 59) -, y tras las también transitorias adaptaciones de Fernando de los Ríos (julio de 1932) y dos años de debates en Cortes, el nuevo ministro de Instrucción Pública y Bellas Artes Filiberto Villalobos González, consiguió la aprobación de un nuevo plan de estudios para el Bachillerato (29 de agosto de 1934) que hacía honor a las inquietudes pedagógicas antes señaladas. En cuanto a la enseñanza de las lenguas vivas este plan era sumamente esperanzador. En primer lugar se estudiaban dos lenguas, el francés durante los primeros cuatro cursos, y un segundo idioma (inglés o alemán) en los dos últimos (de un total de siete cursos). En los exámenes de conjunto los idiomas adquieren un papel extraordinario para lo que era costumbre. En el examen que concedía, tras el quinto curso, el «Certificado de Estudios Elementales de los Estudios Secundarios »- y con el mismo el acceso a las Escuelas Normales, por ejemplo -, habría un ejercicio de francés consistente en una traducción directa e inversa y en la explicación oral de un texto. En cuanto al examen de reválida, tras el séptimo curso, un profesor de idiomas formaría parte del mismo, junto a los habituales de Letras y Ciencias (Calle 1990: 220-223). Tales novedades desaparecerían tras la guerra y tardarían décadas en volver a la enseñanza oficial española.

En lo que se refiere a las orientaciones metodológicas, el cuestionario, publicado el 21 de octubre, aun siendo una solución de compromiso entre tendencias opuestas (Fernández Fraile 1996 : 274), no decepciona :

- Objetivos prácticos y culturales.

- El método directo en el primer curso, cuyo examen sería exclusivamente oral.

- Tendencia más tradicional a continuación (textos literarios seleccionados, traducción, redacción, gramática), pero siempre con una tendencia muy práctica y una preocupación constante por el oral' ${ }^{6}$.

- Progresión gramatical según criterios de simplicidad y de necesidades expresivas.

- Aprendizaje inductivo.

- Se recomienda el uso del gramófono (a partir del tercer curso). 

avanzadas en la enseñanza de las lenguas vivas, pero que tendría una escasa repercusión al no disponer de apenas tiempo para dejar huella. De hecho, los alumnos que empezaron su Bachillerato con este plan lo terminarían con el plan franquista de 1938.

En lo que se refiere al profesorado, tras la decadencia del Museo Pedagógico Nacional, sería la Escuela de Estudios Superiores del Magisterio la encargada de la formación pedagógica del profesorado, y gozaría de gran prestigio e influencia en el ambiente educativo español (García Hoz 1980 : 199). Junto al esfuerzo republicano encaminado a mejorar la formación de los maestros, con la desaparición en 1932 de la Escuela de Estudios Superiores del Magisterio y la creación de la Sección de Pedagogía en la Facultad de Filosofía y Letras de la Universidad de Madrid, los profesores de ciencias y letras se formarían en sus respectivas facultades, con la obligación de cursar dos asignaturas de carácter pedagógico de esa sección. La Sección de Pedagogía tenía como uno de sus fines (Decreto de 27 de enero de 1932), la formación del profesorado de segunda enseñanza y de escuelas normales y habilitaba, a través de un "Certificado de estudios pedagógicos", a los licenciados para oposiciones a cátedras de institutos o escuelas normales. Pero, como tantas otras reformas, este certificado, al igual que el nuevo proyecto de formación del profesorado, quedó en un mero propósito : la primera promoción - dos licenciados - salió en febrero de 1936 y la guerra acabó con esta organización de la facultad (García Hoz 1980 : 178).

En cuanto a la formación específica del profesorado de lenguas vivas, hasta el periodo republicano prácticamente el vacío: apenas el Curso de Ampliación de la Escuela Central de Idiomas de Madrid y una asignatura optativa de literatura contemporánea de lenguas neolatinas en la sección de letras de la Facultad de Filosofía y Letras de Madrid (Fernández Fraile 1996 : 229).

La creación de la Licenciatura de Filología Moderna en 1932 en las universidades de Madrid y Barcelona, y la exigencia de dicha titulación para poder presentarse a las oposiciones a cátedra de instituto a partir de 1935 parecían abrir por fin las puertas a una transformación radical de la formación del profesorado y, con ello, a la enseñanza misma de lenguas vivas. Pero la guerra civil segó de cuajo estas expectativas, puesto que los vencedores de la contienda harían tabla rasa de las reformas republicanas y hasta 1954 no se empezaría a recuperar el tiempo perdido (Calle 1990 : 20-21).

Entre tanto, la situación profesional del profesorado de lenguas vivas seguía siendo muy precaria. Aunque desde 1915 (Decreto de 30 de abril) se exigía el título de Licenciado en Filosofía y Letras para poder acceder a cualquier tipo de puesto docente en los institutos, la realidad era que no se respetaba este requisito y que, al no introducirse modificaciones al respecto en las reformas de 1926 (Calle 1990: 227) la figura del profesor especial (y su papel marginal en los centros) se mantendría durante todo el primer tercio del siglo. Por regla general bastaba con demostrar 'competencia' en la lengua extranjera para impartir clases de la misma. Así pues, hasta el periodo republicano había dos tipos diferentes de profesores de idiomas (en su gran mayoría de francés) en los centros de segunda enseñanza: los catedráticos por oposición, y los profesores ingresados por simple concurso, procedimiento que dio lugar a un reclutamiento muy variopinto y controvertido (Morales Gálvez 2000: 79-80). Para regular la situación de todo este profesorado auxiliar se convocarían unas oposiciones restringidas en 1930. En cuanto al profesorado más cualificado, en 1931 se reformaría el 
sistema de oposiciones a cátedra de instituto, estableciéndose para las de lenguas vivas que los ejercicios se harían en el idioma correspondiente y concediéndose un cierto papel a la formación metodológica de los aspirantes (Fernández Fraile 1996 : 228-229). Al igual que con la formación inicial, estas mejoras tendrían una vigencia tan corta que no llegarían a cambiar significativamente la situación anterior.

También en la década de los treinta se inician los intercambios internacionales de futuros profesores de idiomas, los llamados auxiliares de conversación (Morales Gálvez 2000 : 74). Estos intercambios, que sí se mantendrán tras la guerra con más o menos vicisitudes, jugarán un importante papel en la enseñanza de las lenguas vivas, tanto como complemento de la formación del profesorado, como en la docencia misma, al favorecer con la presencia de estos jóvenes nativos bien formados académicamente (algo que no siempre ocurría con los profesores nativos), la introducción en la clase de la lengua usual y de la civilización extranjera contemporánea.

\section{Franquismo, educación y enseñanza de idiomas}

Durante la guerra, al contrario que en la zona republicana, en la que los proyectos reformadores o revolucionarios de todos los órdenes no se pararon casi hasta el final de la contienda, la educación había ocupado un lugar muy secundario en la España nacional. La nueva educación franquista se comenzaría a configurar a partir del curso 1939-1940, aunque siguiendo algunas bases ya establecidas durante el periodo bélico : fin de la coeducación, depuración del profesorado, control absoluto por parte del poder político y una atención preferente e inmediata a la enseñanza secundaria, en detrimento de la enseñanza primaria (Puelles 1986: 383), plasmada en la Ley Reguladora de los Estudios de Bachillerato de 20 de septiembre de 1938 (BOE del 23), que restablecía una especie de neoclasicismo humanista-cristiano ya muy caduco (Monés 1999 : 18-19). Es decir, una marcha atrás generalizada en todos los ámbitos, provocada primero por la destrucción bélica, el aislamiento internacional y también por la involución ideológica del nuevo Estado, que afectó muy particularmente al sistema educativo y a sus valores. Véase, como ejemplo, el discurso del jefe del Servicio Nacional de Primera Enseñanza, Romualdo de Toledo, en el cursillo para maestros que tuvo lugar en Pamplona el 1 de junio de 1938 (en Mata 1976: 41) :

Y fue señores, el espíritu romántico de aquel 'Emilio' de Juan Jacobo Rousseau, el primer sillar colocado para elevar durante todo el siglo XIX el templo del liberalismo, donde se adoró a la diosa Razón, sustituyendo los principios de la Fe, verdad revelada, en la cual tienen que asentarse fatalmente todos los principios de la educación, aunque se haya querido sustituiría por ese postulado hipócrita del respeto a la conciencia del niño, que nos ha llevado, arrancando desde la más tierna infancia al ciudadano, del Estado laico, a sustituir los prejuicios palabras de Igualdad, Fraternidad y Libertad por el más tirano de los despotismo.

Al mismo tiempo, y para completar la vuelta de hoja, asistimos a la aplicación del 'principio de subsidiariedad', por el que el estado cedía sus responsabilidades a organismos privados, en este caso a la iglesia católica (Tiana 1999 : 35). Hasta tal punto el estado franquista había entregado la educación a los centros privados que incluso se llegó a poner en duda en tela de juicio la existencia misma de los institutos : " Hoy puede pensarse, con sentido práctico, en una posible extinción de los Institutos, sin que se acuse gran alteración en la marcha de la Enseñanza Media »(Frutos $1947: 126)$. 
34 A raíz de la victoria nacional, se producirá una marcha atrás radical hacia la llamada “escuela tradicional decimonónica”, en la que todas las experiencias modernizadoras de la década anterior serán " anatemizadas por extranjerizantes » y revolucionarias (Monés 1999: 30), como preconizaba el editorial anónimo del primer número de la ortodoxa Revista Nacional de Educación (1941:4) :

Quienes se decían depositarios del espíritu tradicional, abandonaron el campo a los enemigos de la Patria, que se adueñaron de todos los resortes del Estado para fraguar e imponer en el orden de la cultura pública el germen de la revolución. La sociedad se encastilló en sus reductos docentes, y no quiso ver que la mala semilla prosperaba en la entraña educativa del Estado (...). La enseñanza oficial, fue coto abierto por la hipocresía liberal a los evangelizadores del marxismo.

A una educación más tradicional, burocrática, conservadora, uniformizadora y poco creativa, se le añadiría una politización continuamente presente, dentro de una ideología e incluso de una escenografía similar a la que se estilaba en los países del eje Berlín-Roma-Tokio. Monés (1999: 19) resume así las características de la educación española en estos años :

- Escasa inversión.

- Rígido control programático e ideológico.

- Renovado papel de la Iglesia como agente educativo.

- Depuración política del profesorado.

- Separación y diferenciación de sexos.

- Enseñanza autoritaria : orden y disciplina, lo que conllevaba una actitud pasiva del alumnado.

En resumen, que durante esta década de los cuarenta, coherentemente con el afán general de destruir todo rastro del pasado reciente ${ }^{7}$, la pedagogía oficial española - la única con voz - aborreció las corrientes pedagógicas renovadoras que habían cobrado un importante auge en la España republicana.

De las verdaderas intenciones del nuevo Estado en materia de educación da cumplida cuenta el propio ministro de Educación Nacional, Pedro Sainz Rodríguez, en su discurso con motivo de la sesión de clausura de los cursillos de orientadores nacionales de primera enseñanza, celebrados en Pamplona en 1938. Tras citar un discurso de Fernando de los Ríos, en el que éste señalaba que los discípulos de Giner de los Ríos se habían "injertado" en las principales instituciones educativas españolas, como la Escuela Superior del Magisterio, la Junta para la Ampliación de Estudios e Investigaciones Científicas, la Escuela de Criminología o la Residencia de Estudiantes, y que estos eran los gérmenes que habían posibilitado el advenimiento de un régimen nuevo, el ministro concluía, entre risas y aplausos, que aquellas palabras « eran para nosotros tan preciosas como si fuesen un mapa donde nos hubieran señalado las fortificaciones que tenemos que bombardear » (en Molero 1991 : 463).

Da pavor leer esas líneas y se comprende demasiado bien lo que les esperaba a los educadores 'rojos' que fueran hechos prisioneros; y se comprende mejor que los que pudieran partieran al exilio. En todo caso, los objetivos estaban claros para los triunfadores de la contienda y así, el mundo de la educación y la cultura serían de los más castigados por la represión franquista, porque «el rigor se endureció conforme fueron pasando los meses, y así en la orden ministerial de 18 de marzo de 1939 se considera causa suficiente de depuración la 'pasividad evidente de quienes pudieron haber cooperado al triunfo del Movimiento'« (Puelles 1986 : 368). Pasarían largos años 
antes de que la enseñanza española, en todos sus niveles, lograra empezar a sobreponerse del golpe.

En cuanto a la enseñanza de los idiomas, nada podrá salvarse de la vorágine revanchista. A la promulgación en plena guerra civil de la nueva Ley Reguladora de los Estudios de Bachillerato (20 de septiembre de 1938), que cortaba las alas modernizadoras republicanas, seguirán la supresión inmediata de los recién creados estudios de Filología Moderna y el olvido de todas las reformas tendentes a elevar el papel y la cualificación del profesorado de idiomas.

\section{Conclusiones}

Con la llegada de la República se habían abierto, también para la enseñanza de las lenguas vivas, perspectivas esperanzadoras: un nuevo papel de los idiomas en el sistema educativo, la renovación de los métodos de enseñanza y su adaptación a las corrientes más modernizadoras, la especialización de la formación inicial universitaria, un nuevo concepto de la formación psicopedagógica del profesorado y la consiguiente revalorización de su situación profesional. Con la derrota "roja" se frustraron bruscamente los intentos republicanos de adaptar la pedagogía española a las corrientes vanguardistas europeas $\mathrm{y}$, a la vuelta de la guerra civil, en consonancia con un periodo reconocido como de los más ominosos de la historia de España, de todas aquellas iniciativas no quedaría más que un enorme vacío. Ilusiones perdidas.

BIBLIOGRAFÍA

\section{Bibliografía}

ANÓNIMO. 1941. « Editorial ». Revista Nacional de Educación $1: 4$.

BOHLEN, Adolf. 1963. Metodología de la enseñanza de los Idiomas Modernos. Madrid : Dirección General de Enseñanza Media.

BUREAU INTERNATIONAL D'ÉDUCATION. 1937. L'enseignement des Langues Vivantes (D'après les données fournies par les Ministères de l'Instruction Publique). Genève : BIE.

CALLE CARABIAS, Quintín. 1990. La enseñanza oficial de idiomas en España. Por una redefinición de la formación teórica del profesorado. Málaga, Málaga : Universidad de Málaga, Tesis Doctorales/ Microficha.

CANITO, Enrique. 1952. «Antonio Tovar y la renovación de los estudios universitarios ». Ínsula $84: 8$.

CASANOVA, Julián. 2009. « El castigo a los vencidos ». El País, 1 de febrero de 2009, 25.

CASTRO, Américo. 1921. « La enseñanza de las lenguas modernas ». Boletín de la Institución Libre de Enseñanza 733 : 119-121. 
CUESTA ESCUDERO, Pedro. 1994. La escuela en la reestructuración de la sociedad española (1900-1923). Madrid : Siglo XXI de España Editores S. A.

DOMÍNGUEZ DOMÍNGUEZ, Consuelo. 1999. La reforma educativa de la II ${ }^{\underline{a}}$ República : Huelva, 1931-1936. Huelva : Diputación de Huelva.

FERNÁNDEZ FRAILE, María Eugenia. 1996. La enseñanza-aprendizaje del francés como lengua extranjera en España : 1767-1936. Granada : Universidad de Granada, Tesis doctorales/Microfichas.

FRUTOS CORTÉS, Eugenio de. 1947. « Perspectivas de los Institutos Nacionales de Enseñanza Media ». In aa.vv. Cuestiones de Enseñanza Media, Madrid : CSIC, Instituto San José de Calasanz, 125-137.

FUSI AIZPURÚA, Juan Pablo. 1998. « En los comienzos de un nuevo reinado » in aa. vv., Memoria del 98. De la guerra de Cuba a la Semana trágica. Madrid : El País/Aguilar, 325-330.

GAMBOA SEGGI, G. 1955. « Algunas consideraciones sobre la situación y enseñanza de las lenguas vivas en Francia ». Revista Española de Pedagogía XIII, 51 : 205-219.

GARCÍA DE CORTÁZAR, Fernando y GONZÁLEZ VESGA, José Manuel. 1994. Breve Historia de España, Madrid : Alianza Editorial.

GARCÍA HOZ, Víctor. 1980. La educación en la España del siglo XX. Madrid : Rialp.

MARTÍNEZ DE BLANCO, María. 1962 ? « La Federación Internacional de Profesores de Lenguas Vivas ", in Luís Grandía Mateu et al. Nuevos Métodos en la enseñanza de idiomas. Madrid : Dirección General de Enseñanza Media, 135-142.

MATA, Marta. 1976. «La Práctica de la Formación y de la Pedagogía ». Cuadernos de Pedagogía 3 : $40-42$.

MONÉS I PUJOL-BUSQUETS, Jordi. 1999. « La educación en España en el siglo XX ». Cuadernos de Pedagogía 286 : 12-33.

MOLERO PINTADO, Antonio. 1991. La Educación durante la Segunda República y la Guerra Civil (1931-1939). Madrid : Ministerio de Educación y Ciencia.

MORALES GÁLVEZ C. et al. 2000. La enseñanza de las lenguas extranjeras en España. Madrid : Ministerio de Educación, Cultura y Deporte, Centro de Investigación y Documentación Educativa, en http://www.mec.es/cide/publicaciones/textos/inv2000elee/inv2000elee.htm [consultado en diciembre 2002].

MOSSÉ, Fernand. 1960 ? « Préface ", in François Closset. Didactique des langues vivantes. Buts, méthodes, procédés et matériel de cet enseignement. Bruxelles-Paris : Marcel Didier, 5-7.

PUELLES BENÍTEZ, Manuel de. 1986. Educación e ideología en la España contemporánea (1767-1975). Madrid : Labor.

PUREN, Christian. 1998. Histoire des méthodologies de l'enseignement des langues. París : Nathan CLE International.

ROGER, Juan. 1953. « La enseñanza de las lenguas vivas en el extranjero ». Revista de Educación 9 : 27-31.

ROMERO MUÑOZ, Antonio. 1998. La pugna por el control y el desarrollo de la escuela primaria en Huelva en la crisis de la Restauración. Huelva : Diputación Provincial.

SÉCHEHAYE, Albert. 1921. «The principles of language study by Harold E. Palmer ». Revue de l'Enseignement des Langues Vivantes XXVIII : 461-464. 
SICART, Pablo. 1947. Metodología Científica de las Lenguas Vivas. Madrid : Ministerio de Educación Nacional.

TIANA FERRER, Alejandro. 1999. «El proceso de escolarización ». Cuadernos de Pedagogía 286 : 34-36.

UTANDE IGUALADA, Manuel. 1982. « Un siglo y medio de Segunda Enseñanza (1820-1970) ». Revista de Educación XXX, $271:$ 7-42.

\section{NOTAS}

1. «Este derecho que tiene la Iglesia sobre la enseñanza se fundamenta en que si Ella es quien únicamente posee la verdad, Ella es la única autorizada a dirigir la enseñanza. Y también se basa en que el fin religioso es el fin supremo del hombre. La enseñanza y educación han de estar íntimamente ligados a ese supremo fin salvador. Y si la Iglesia, precisamente, es la que encarna este fin religioso, a ella compite [sic], pues, la dirección de la enseñanza » (Cuesta Escudero 1994 : 189).

2. «La reforma [instrucciones directas francesas] de 1902 abrió las puertas a las esperanzas y ambiciones de esta enseñanza nueva : puesta a igualdad con la enseñanza de las demás asignaturas, se trataba no sólo de leer en lengua extranjera, sino de hablarla y escribirla. Salto prodigioso, que molestó... ¡naturalmente!, a los defensores de las lenguas muertas o clásicas, el latín sobre todo. Lucha encarnizada... » (Gamboa 1955 : 208).

3. En España, la reforma de Romanones (1901) estipulaba en 150 el número máximo de alumnos por clase en los Institutos generales y técnicos (Calle $1990: 204$ ).

4. Desde 1911, con la creación de la Escuela Central de Idiomas y en su $4^{\circ}$ curso, se ofrecían cursos de perfeccionamiento para profesores de lenguas vivas.

5. En realidad, la situación de la educación en España era en sí catastrófica : a principios de siglo la tasa de analfabetismo era del 75 \% de la población (Fusi 1998), y hacia 1920 la cifra de analfabetos rondaba, según las dudosas estadísticas de la época, entre el 50 y el 35\% (García Hoz 1980 : 37).

6. Se trata de un tipo de eclecticismo muy habitual en aquellos años : no se integran los principios (los directos y los tradicionales), sino que se yuxtaponen en el tiempo (Puren 1988 : 194-195).

7. El 9 de febrero de 1939, Franco había firmado la vengativa Ley de Responsabilidades Políticas (Casanova 2009).

\section{RESÚMENES}

A partir de 1931 un esfuerzo de modernización llega a todos los rincones de la España republicana, incluido el pequeño micromundo de la enseñanza de las lenguas vivas : reforma de la enseñanza secundaria de 1934, nuevo papel de las lenguas vivas en el sistema educativo, creación de los estudios de Filología Moderna, aparición del método directo en la enseñanza oficial... Sin embargo, la guerra civil se llevará consigo súbitamente esos aires modernizadores. 
From 1931 in Republican Spain an effort to modernize reaches every corner of Spanish life, including the small microworld of the teaching of modern languages : reform of secondary education in 1934, the new role of modern languages at education system, building studies of Modern Philology, appearance of the direct method in formal education... However, civil war will get suddenly these winds modernizers.

\section{ÍNDICE}

Mots-clés: enseignement des langues en Espagne, XXe siècle, république espagnole, réforme de l'enseignement, guerre civile, franquisme et éducation

Keywords: language teaching, Spain, XXth century, spanish Republic, reform, spanish civil war, Franco, education

\section{AUTOR}

\section{FRANCISCO JOSÉ MORALES GIL}

Universidad de Huelva 